\title{
The Influence of Dynamic Signs on Cyclists' Braking Rates: A Systematic Study Using Immersive Virtual Reality
}

\author{
Hiroshi Watanabe ${ }^{*}$, Nana Ito ${ }^{1}$, Hiroyasu Ujike, Reiko Sakata ${ }^{2}$, Munetaka Nishihira², \\ Tsutomu Matsubara ${ }^{2}$
}

${ }^{1}$ Human Informatics Research Institute, National Institute of Advanced Industrial Science and Technology, Tsukuba, Japan ${ }^{2}$ Industrial Design Center, Mitsubishi Electric Corporation, Kamakura, Japan

Email: ^h.watanabe@aist.go.jp

How to cite this paper: Watanabe, H., Ito, N., Ujike, H., Sakata, R., Nishihira, M., \& Matsubara, T. (2017). The Influence of Dynamic Signs on Cyclists' Braking Rates: A Systematic Study Using Immersive Virtual Reality. Psychology, 8, 1301-1313. https://doi.org/10.4236/psych.2017.89084

Received: May 22, 2017

Accepted: July 11, 2017

Published: July 14, 2017

Copyright (ङ 2017 by authors and Scientific Research Publishing Inc. This work is licensed under the Creative Commons Attribution International License (CC BY 4.0).

http://creativecommons.org/licenses/by/4.0/ (c) (i) Open Access

\begin{abstract}
Dynamic signs are signs that incorporate movement and are projected from a vehicle toward pedestrians or on the wall or floor of a public facility. Projection-based signage systems that are easy to install and move are becoming increasingly practical as a result of recent improvements in projector technology, and there is a need to accumulate knowledge on such signs and their interactions with people from an ergonomic perspective. This paper studies dynamic signs projected from parked cars (these signs warn that the car is about to reverse or open a door) with the goal to clarify the influence of these dynamic signs on the braking behavior of cyclists riding near the cars. In an experiment, we placed several parked cars in an immersive virtual reality space and created a system that allowed participants to move through the virtual space by steering a real bicycle. As participants rode past the row of parked cars, they took evasive actions to avoid a collision by actually operating the brake and handlebar in response to signs projected from cars warning that the car was about to reverse or open a door. For the experimental conditions, we looked at participant age groups, the method used to display the signs, and the timing of displaying the sign. The results suggest that the various experimental conditions produce different effects, and our discussion focuses on brake selection rates as a measure of cyclists' responses to events.
\end{abstract}

\section{Keywords}

Dynamic Signs, VR, Braking Rates, Risk Assessment, Effect of Aging, Cognitive Decline, Simulator Sickness

\section{Introduction}

Accidents involving vehicles and pedestrians can occur while cars are parked, 
and when cars are in motion. According to a study by the Institute for Traffic Accident Research and Data Analysis (2016), about 6200 fatal accidents involving pedestrians and automobiles took place in car parks between 2010 and 2014 in Japan. Reports characterize the circumstances surrounding these accidents as including many cases with pedestrians over the age of 65 when a car is reversing, or pedestrians under the age of six when a car starts moving forward. At the same time, there are also reports that more than half of the accidents involving stationary vehicles in 2014 had doors opening as a factor (2325 accidents out of a total of 3800) (Institute for Traffic Accident Research and Data Analysis, 2015). Cyclists were particularly susceptible to such accidents, accounting for $67 \%$ of cases, with motorcycles and mopeds accounting for a further $19 \%$. The party at fault in door-opening-related accidents is not necessarily the driver; it can also be any passenger, including children.

One possible solution for preventing such accidents is for parked vehicles to display signs warning pedestrians and cyclists of their impending actions. In this case, attention is drawn to the stationary vehicle and the meaning of the warning sign is conveyed, allowing the pedestrian or rider to take evasive action. For this use case, the design of such signs must consider the subsequent evasive action as well as how readily the signs can be noticed and understood.

Our previous work used real video footage of dynamic sign information warning that a door will open being projected onto the road to obtain data on the occurrence of observers' brake selection behavior, from the perspective of subjective reports and the time taken to make a decision (Sakata, Matsubara, Watanabe, Ito, \& Ujike, 2016). The results suggested that both the timing and animation of the sign influence the awareness of the sign and the evasive action taken by the cyclist (including the type and the time taken to reach a decision).

This study builds on the earlier work in three ways: 1) we add scenarios in which cars move in reverse as well as car doors opening; 2) we obtain data under consistent experimental conditions by using an immersive virtual reality device to arrange vehicles (rendered using three-dimensional computer graphics) and control how the signs are displayed; and 3) we obtain quantitative data on brake and steering operations by having participants used a real bicycle to move through the virtual reality space.

\section{Methods}

\subsection{Participants}

Bicycle-riding behavior under ordinary conditions draws on various cognitive functions such as visual attention, time perception, spatial perception, motor control for equipment operation, and decision making. It is thus important to clarify the effect of aging on that behavior. This is of particular concern in Japan because dementia rates have been increasing rapidly with the aging of the population, as is happening in other countries (Miyanaga, 1997). When looking at the rate of dementia by age in Japan, the rate of dementia among people less than 40 
years old is $0.2 \%$, among those $40-59$ years old is $2 \%$, and among those $60-79$ years old is $16 \%$ (Ministry of Health, Labour and Welfare, 2009). This epidemiological fact suggests that the characteristics of cognitive function change with aging; because of this, we grouped participants by age, with divisions at 40 and 60 years.

A total of 100 participants were divided into three groups: 20 - 39 years old (n $=34), 40-59$ years old $(\mathrm{n}=35)$, and $60-79$ years old $(\mathrm{n}=31)$. We recruited persons with normal vision, hearing, and walking ability who ride a bicycle in the course of their daily life, offering financial compensation for participation. On the day of the tests, we verified that there were no impediments to participation, using a questionnaire about their physical condition on the day, as well as using Landolt rings from a distance of $5 \mathrm{~m}$ and the Stereo Fly Test (Stereo Optical Co., Inc.) to examine visual acuity and stereo vision. No participants had any prior knowledge of the content of the test. The content of the test was approved by the Ergonomics Committee of the National Institute of Advanced Industrial Science and Technology (AIST), and we obtained written consent to participate in the test from each participant. Table 1 shows the number of men and women in each age group and visual acuity scores. Note that the results of the visual acuity exam using Landolt circles are expressed differently in Japan than they are in Europe and North America. For example, the Japanese visual acuity scores $0.5,1.0$ and 2.0 would be expressed as 20/40,20/20 and 40/20, respectively, in Europe and North America.

\subsection{Pretest Examinations}

We examined the following items before the tests using the virtual reality device: 1) the Cognitive Aging Test developed by AIST (AIST-CAT), and 2) the MiniMental State Examination (MMSE) (Folstein, Folstein, \& McHugh, 1975). Both of these tests evaluate cognitive functioning, but the MMSE is used as an index of cognitive impairment and has been shown to be inappropriate for functional assessment in healthy elderly populations, the majority of whom score full points for sensory faculties (Suto, Kumanda, Kitajima, \& Sato, 2008). Accordingly, we chose to use this test in conjunction with AIST-CAT, which was developed to measure cognitive decline due to aging in healthy elderly people. Table 1 shows the results of these two cognitive tests for each age group.

\subsection{VR Experiments}

This section describes the test, which involves moving through a virtual reality space using a real bicycle. This test was the principal focus of this paper.

Device. Test video images were projected from 4000-lm projectors (NPM402XJD by NEC) onto four 3-m square screens placed to the left and right of the viewer as well as to the front and on the floor, using the CAVE immersive virtual reality device (produced by the University of Illinois). Participants viewed these projections through glasses that had been polarized with the circular light polarization method (Figure 1). The position of participants' heads was meas- 
Table 1. Participants characteristics and pretest results by age group.

\begin{tabular}{ccccc}
\hline & & $\mathbf{2 0}-\mathbf{3 9}$ years old & $\mathbf{4 0}-\mathbf{5 9}$ years old & $\mathbf{6 0}-\mathbf{7 9}$ years old \\
\hline \multirow{2}{*}{ Men } & $N$ & 18 & 16 & 16 \\
& Distance vision & $1.2 \pm 0.4$ & $1.2 \pm 0.3$ & $1.1 \pm 0.2$ \\
& Stereo vision & $12.7 \pm 0.8$ & $12.0 \pm 1.8$ & $10.9 \pm 2.4$ \\
& Cognitive test 1 & $141 \pm 7.6$ & $139 \pm 7.1$ & $121 \pm 14.8$ \\
& Cognitive test 2 & $29.1 \pm 0.9$ & $28.3 \pm 1.2$ & $27.8 \pm 1.9$ \\
Women & $N$ & 16 & 19 & 15 \\
& Distance vision & $1.1 \pm 0.2$ & $1.3 \pm 0.4$ & $1.0 \pm 0.3$ \\
& Stereo vision & $12.8 \pm 0.4$ & $12.6 \pm 1.2$ & $11.6 \pm 2.4$ \\
& Cognitive test 1 & $143 \pm 4.7$ & $137 \pm 14.7$ & $127 \pm 17.8$ \\
& Cognitive test 2 & $29.3 \pm 0.8$ & $28.2 \pm 1.4$ & $28.2 \pm 2.0$ \\
\hline
\end{tabular}
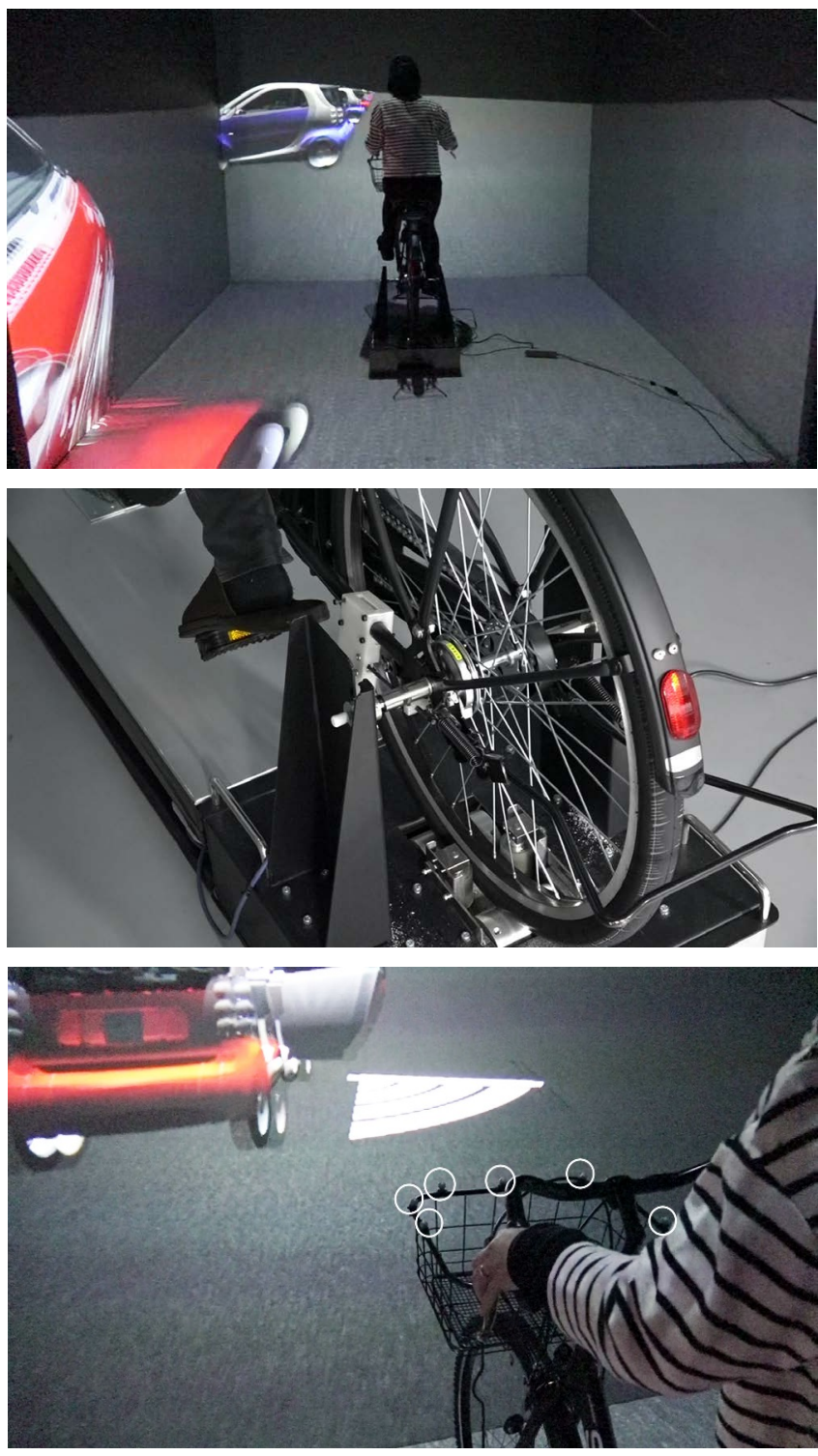

Figure 1. (Top) The cycleStreet system installed in the CAVE. (Middle) The cycleStreet roller for detecting the rotation speed of the back wheel. (Bottom) The markers (highlighted by circles) for measuring the bicycle's direction of movement. 
ured without contact at a sampling rate of $120 \mathrm{~Hz}$ using the Vicon system (Vicon) and position data was transmitted to the CAVE system via an Ethernet connection (average height of top of head was about $154 \mathrm{~cm}$ ). This information was used to correct rendering distortions based on the position of the test participant's head. Movement through the virtual reality space was achieved via the Cycle Street City Multi-Purpose Virtual Cycling System (2016) (Flovel, 2016). With this system, speed data are obtained from a sensor on the back wheel of the bicycle that measures the rotation speed, while braking data are obtained from the length of the brake wire. The maximum velocity of the bicycle was restricted to $10 \mathrm{~km} / \mathrm{h}$ for the simulation to reduce the differences between subjects. The direction of movement was determined by converting position data for the $\mathrm{Vi}$ con system marker attached to the front of the bicycle to a quaternion in the three-dimensional space.

Procedure. Participants were asked to control the bicycle so as to ride to the right of parked cars that were placed at irregular intervals along a straight line via computer graphics. The data for the parked cars was all derived from $3 \mathrm{D}$ models (by CG Databank) of the "Smart" make of car, which is $1.51 \mathrm{~m}$ wide and $2.5 \mathrm{~m}$ long. The average spacing between parked cars was $4.5 \mathrm{~m}$.

As participants rode through the space, a car door might open or a car might start reversing. These events were accompanied by the display of warning signs, and participants were asked to either stop or avoid collision at their own discretion. Participants had no prior knowledge about which cars might initiate these events. Cars whose doors opened were parked parallel to the direction of travel, whereas reversing cars were parked perpendicular to the direction of travel. However, all cars were of the same shape, painted either red or blue at random, and there were no clues indicating the relation between cars and events.

The signs indicating a car door about to open were $1.9 \mathrm{~m}$ wide and $1.9 \mathrm{~m}$ high $\left(3.61 \mathrm{~m}^{2}\right)$, while the dimensions of the signs indicating a car about to reverse were $2.4 \mathrm{~m}$ wide by $1.5 \mathrm{~m}$ high $\left(3.60 \mathrm{~m}^{2}\right)$. The timing of sign display was either $3.2,4.6,5.9$ or $7.2 \mathrm{~s}$ before the door opening or car reversing event. Our previous work (Sakata et al., 2016) used real video footage of dynamic sign information and employed timings of $0.5,3$ and $5 \mathrm{~s}$. However, during a preliminary phase of this study, we found that shorter values were more likely to surprise participants and lead them to engage in sudden and hazardous handling and braking. We therefore added a margin of $2.7 \mathrm{~s}$ to the shortest time $(0.5 \mathrm{~s})$ and set timings at intervals of $1.3 \mathrm{~s}$ to generate 4 timings.

Two types of sign were prepared: animated and flashing. The duration of the frames making up each of the two display methods were as follows. When animated, both the door opening sign and the reversing sign consist of five frames displayed as a loop, with the first and last frames displayed for $0.3 \mathrm{~s}$ each and the intermediate frames displayed for $0.13 \mathrm{~s}$ each. When flashing, both the door opening sign and the reversing sign consist of two frames, each displayed for 0.5 s. These parameters are chosen because they agree with those of a prototype projection system that some of the authors are developing. These settings result 
in both conditions (animated and flashing) having roughly the same frequency of $1 \mathrm{~Hz}$ (Figure 2), and this value is consistent within the range of $1-2 \mathrm{~Hz}$ specified by Japanese law.

To summarize, there are a total of 16 different stimulatory conditions: two events (door opening and car reversing), four display timings (3.2, 4.6, 5.9 and $7.2 \mathrm{~s}$ ), and two display methods (animated and flashing). Each combination was presented three times over the course of the test, so that participants were asked to make a total of 48 decisions split over 12 sessions. Each session lasted about 4 min, so that the total test time was less than $4 \mathrm{~h}$, including breaks.

We also monitored simulator sickness via a questionnaire, and participants who experienced extreme symptoms were allowed to withdraw. Participants filled out a Simulator Sickness Questionnaire (SSQ) (Kennedy, Lane, Berbaum, \& Lilienthal, 1993) before the start of the first session. For participants who felt sick during the experiment and were unable to continue, we stopped the experiment immediately and asked the participant to fill out the SSQ again. Participants who completed all 12 sessions filled out the SSQ after the end of the final session. We first adopted this procedure after 34 participants had already completed the experiment, so only 66 participants filled out SSQs.

\section{Results}

\subsection{Visual Acuity}

We performed analysis of variance on the scores for the 5-m visual acuity exam using Landolt circles and the Stereo Fly stereo vision test (conducted as pretest examinations), using age group (20 - 39 years old, 40 - 59 years old, or 60 - 79 years old) and gender as the between-subjects factors. The two conditions did not have any significant main effects in either test (5-m visual acuity exam: age group $\mathrm{F}(2,94)=1.13, p>.05$; gender $\mathrm{F}(1,94)=0.13, p>.05$; stereo vision test: age group $\mathrm{F}(2,94)=1.90, p>.05$; gender $\mathrm{F}(1,94)=1.94, p>.05)$, indicating no differences in the visual acuities of participants.

\subsection{Cognitive Ability}

Two tests were conducted to assess the cognitive abilities of the participants.

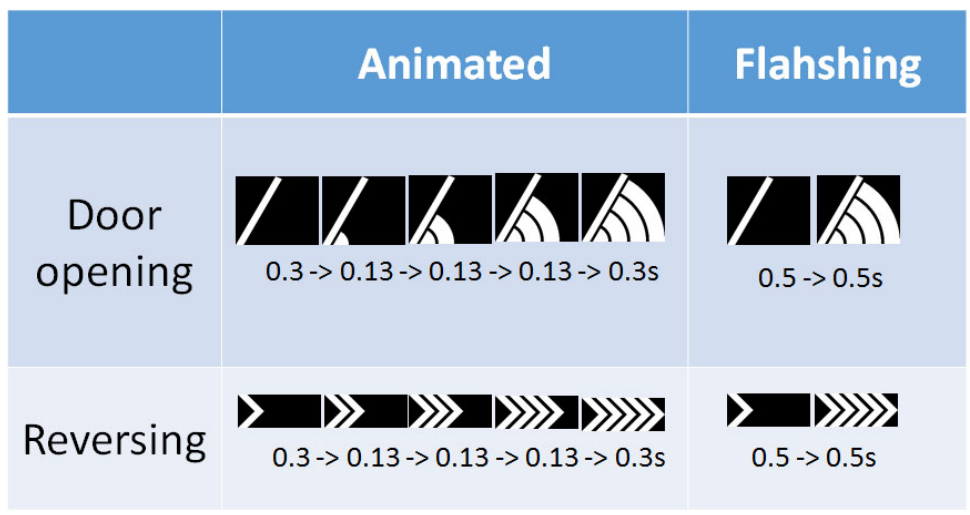

Figure 2. Methods for displaying dynamic signs. 
AIST-CAT consists of five tasks (checking target stimuli, writing mirror letters, switching tasks, visual searches, and activity sequences), and we calculated scores for each individual as the total of the scores for each of these tasks. The MMSE test consists of 11 questions with a maximum score of 30 points, and assesses five areas: orientation, registration, attention and calculation, recall, and language (Folstein, Folstein, \& McHugh, 1975). We calculated scores for each individual as the total of the scores for the 11 questions. Analysis of variance on the scores for these tests, taking age group and gender as the between-subjects factors (in the same way as for visual acuity), found significant main effects for the age group condition in both tests (AIST-CAT: $\mathrm{F}(2,94)=8.26, p<.01$; MMSE: $\mathrm{F}(2,94)=5.10, p<.01)$. When we examined the difference between groups using post hoc multiple-comparison tests, the scores for the $60-79$ year old age groups were significantly lower for both tests. Despite this difference, the average AIST-CAT scores for the elderly group in this experiment were relatively high (123.69 for men and 113.93 for women) compared with the typical score of 103.55 for the elderly in general (Adachi, Harada, Suto, Kumada, \& Fujiwara, 2014). The same trend was evident in the MMSE scores, with the average scores for both men (27.44) and women (24.53) exceeding the 24-point cut-off for categorization as neurologically normal. Accordingly, although we did find a numerically significant difference between age groups, the older age group does not appear to consist of members that would be classed as cognitively impaired.

\subsection{Withdrawal due to Visually Induced Motion Sickness}

The data for the 66 participants who filled out the SSQ was categorized into two classes: 58 participants who completed all sessions and eight participants who dropped out. The SSQ consists of 16 questions on simulator sickness and participants responded to questionnaires in four-option multiple choice format. During analysis, the 16 items are classified using four subcategories ("Nausea," "Oculomotor," "Disorientation," and "Total"). Final scores are obtained by multiplying the raw scores for each subcategory by different factors ( 9.54 for Nausea, 7.58 for Oculomotor, 13.92 for Disorientation and 3.7 for Total). Total scores for the 3 (of 66) participants who completed all sessions were over 55, and we omitted these subjects' data in this section, because it seems likely that they forced themselves to complete sessions (Balk, Bertola, \& Inman, 2013).

Figure 3 shows the average SSQ scores for all participants, participants who completed all sessions, and participants who dropped out.

We also conducted analysis of variance taking completion of all 12 sessions as the between-subjects factor, and the subcategory scores in each group as within-subject factors. The results of the analysis showed significant main effects for both completion $(\mathrm{F}(1,61)=28.60, p<.01)$ and subcategory scores $(\mathrm{F}(3,183)=$ $28.07, p<.01)$. These results show that participants who dropped out clearly felt simulator sickness.

Significant interactions between these main effects were also shown, and so we conducted further examination of the simple main effects, which indicated sig- 


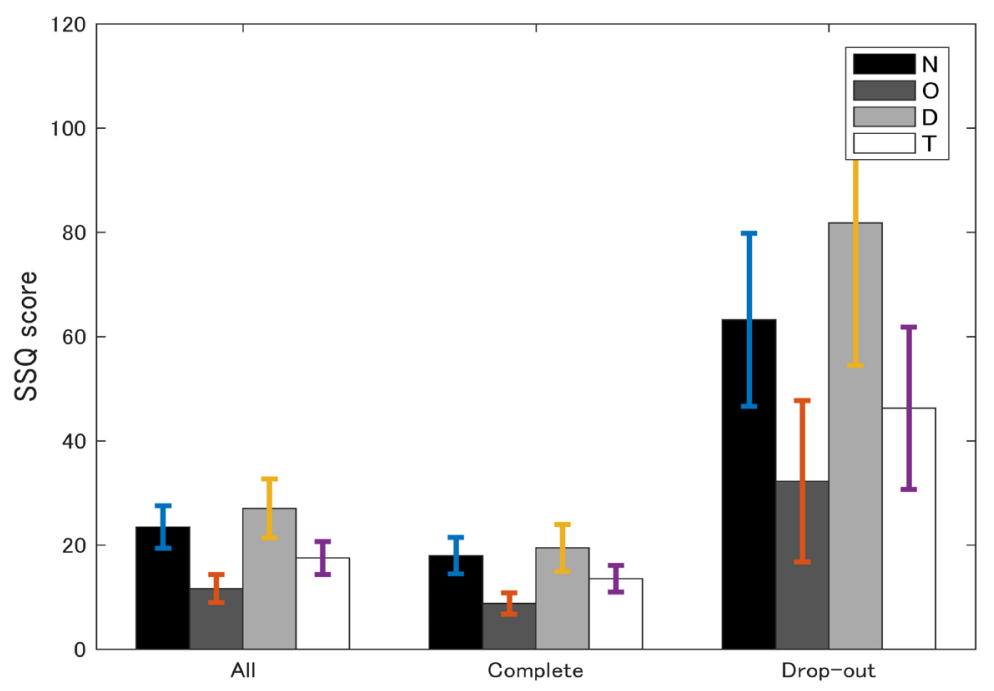

Figure 3. Average SSQ scores for all participants, those who completed all sessions, and those who dropped out.

nificant differences between subcategories in the responses of the drop-out group $(\mathrm{F}(3,183)=14.81, p<.01)$. Multiple comparisons based on the Ryan-Einot-Gabriel-Welsch method showed significant differences between all pairs of subcategories.

Exposure time for VR, traveling distance in VR and total scores on the SSQ for the completed (resp., dropped-out) group are $28.45 \mathrm{~min}(13.11 \mathrm{~min}), 4.74$ $\mathrm{km}(2.19 \mathrm{~km})$, and $10.02(46.25)$, respectively.

\subsection{Evasive Actions While Riding through the Virtual Reality Space}

Next, we discuss the effect on evasive behavior of signs warning of vehicle actions, displayed while participants ride a bicycle through the virtual reality space. This behavior is the main concern of this paper. Figure 4 shows one session's worth of data for one participant as an example of the results we obtained. The top graph in this figure shows the length of the brake wire, and the bottom graph shows the angle of the handlebars. The horizontal axis shows the time (in minutes) elapsed since the start of the test, while the vertical lines indicate the times when warning signs were displayed. The points on each waveform that have been highlighted with circles are the singularities in the brake or handlebar state that occur immediately after the signs are displayed, as automatically detected by a signal processing package (Matlab by MathWorks). Note that the brake waveform patterns immediately after the start of the session and immediately before the end of the session are dummy data for the start and end of the test, and are not associated with the participant's responses.

The discussion below focuses on the brake responses, which occur prior to the handlebar operations. As discussed above, each of the 16 sets of stimulus parameters was presented three times. Thus, participants' possible responses can be quantified as brake selection rates of $0 \%, 33.3 \%, 66.6 \%$, or $100 \%$ for each condition. Figure 5 shows the average values calculated from all participants' res- 

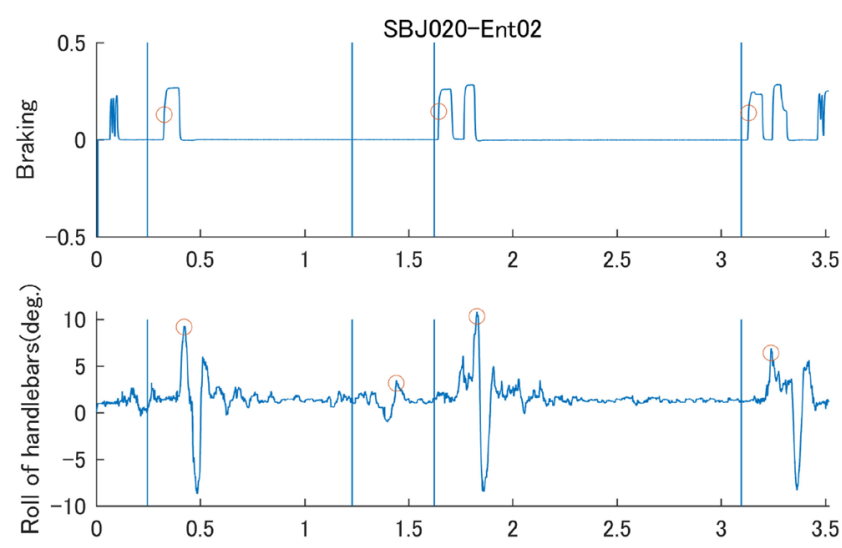

Figure 4. An example of braking and handlebar operations in response to signs. See main text for details.

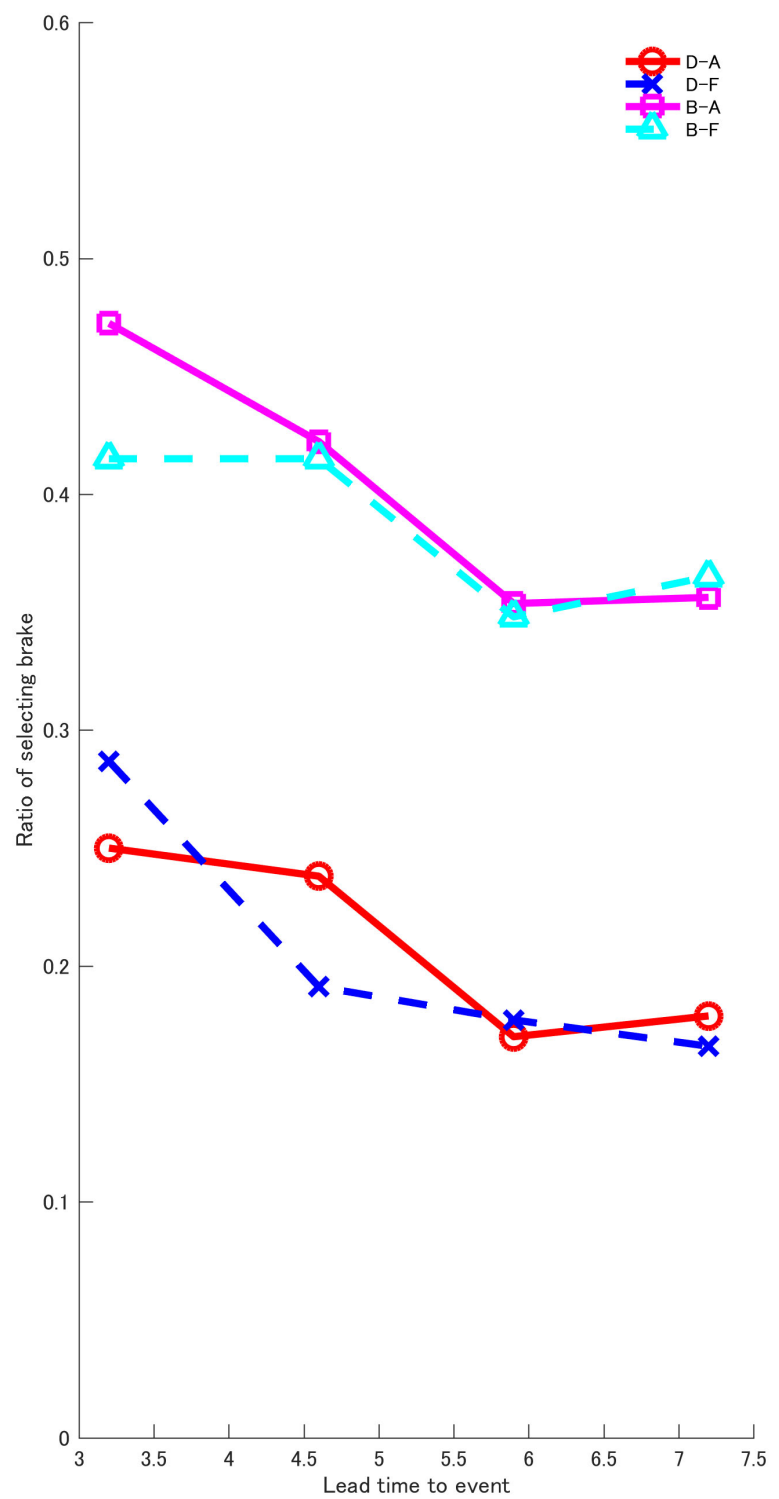

Figure 5. Brake selection rate trends according to display timing (average for all participants). 

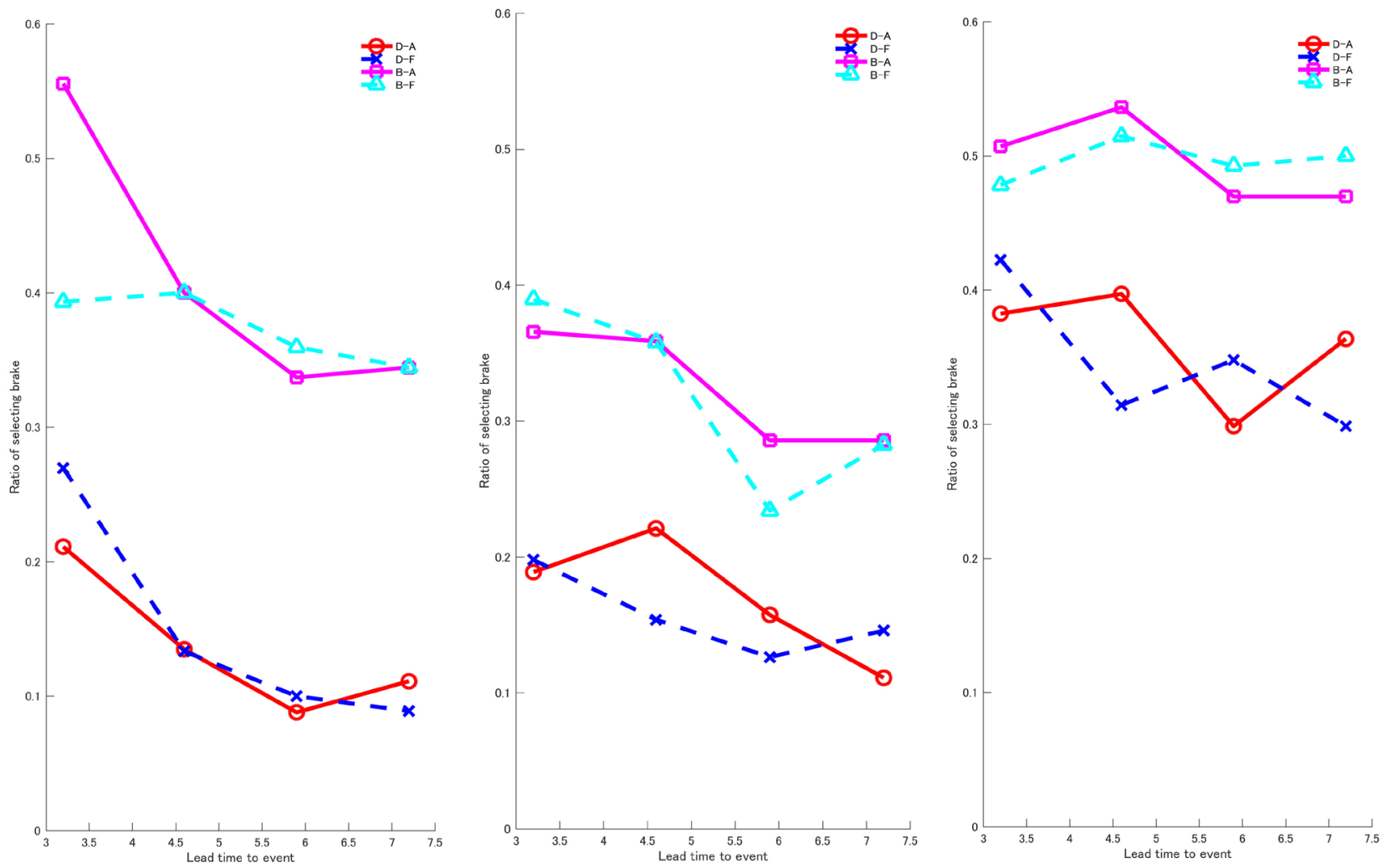

Figure 6. Brake selection rates by age group. From left to right: 20 - 39 years old, 40 - 59 years old, and 60 - 79 years old. 
contrast, the timing of when the signs were displayed resulted in significant differences only for the 20 - 39 years and $40-59$ years age groups $(\mathrm{F}(3,87)=9.56, p$ $<.01$ and $\mathrm{F}(3,87)=4.54, p<.01$, respectively), suggesting older people have a constant brake response rate regardless of the timing $(\mathrm{F}(3,60)=1.65, p>.05)$. Significant interactions were also apparent between the three conditions in the 20 - 39 years group $(\mathrm{F}(3,87)=4.45, p<.01)$. Follow-up testing indicated that for warning signs displayed $3.2 \mathrm{~s}$ before a car reversed, animated signs had significantly higher brake selection rates $(\mathrm{F}(1,232)=16.47, p<.01)$. This matches the pooled result, suggesting that the pooled result reflects the response characteristics of the younger age group.

\section{Discussion}

We have validated the effect on brake selection rates for different age groups of the following parameters: vehicle behavior, dynamic characteristics of the sign warning of imminent behavior, and the timing of displaying the warning sign. The test results showed that brake selection rates are higher when cars reverse, for all ages. This is likely because all age groups are aware that this type of action by a vehicle can cause a serious accident. Interestingly, the $60-79$ years group had a higher brake selection rate in response to car doors opening than other age groups did, and the brake selection rate was not affected by the timing of when the sign was displayed. That is, it seems that this group's first response is to slow or stop when they perceive a sign warning of an imminent event. Aging affects a wide range of cognitive and behavioral characteristics, from a decline in sensory responses at the input stage (Shiota \& Ikeda, 2008) such as lower range of accommodation, lower retinal illuminance, more light scattering, and slower scotopic adaption (Jackson, Owsley, \& McGwin, 1999; Ujike \& Sagawa, 2003) through to longer response times at the behavioral level. As a result of their self-perception of this kind of decline in their sensory and physical capabilities, it seems likely that older people pay greater attention to their surroundings and increasingly select cautious actions. This kind of psychological process is referred to as "hazard perception" in modern risk theory (Brown \& Groger, 1988). In contrast, the 20 - 39 years group shows a tendency to switch between steering and braking, according to the timing of when the sign is displayed. The decision to halt the bicycle upon sensing danger is correct in and of itself, but panic braking can also lead to a dangerous halt, depending on the speed, mass, and road conditions. Hence, it may be necessary to carefully consider whether cars should constantly display signs warning of their impending actions. In some circumstances, there may be a need for sensing technology to detect the surrounding circumstances or the presence of an approaching pedestrian, bicycle, or motorbike.

Note that, in general, signs being animated or flashing seemed to have little effect on the brake selection rate. This result could open up a diverse range of options for sign design. In the process of deliberating more effective methods for displaying signs and establishing standardized guidelines, we anticipate that it 
will be important to collect data under a wide range of conditions.

In summary, we discussed the influence of dynamic signs warning of the behaviors of a parking vehicle on the braking responses of cyclists riding in the VR space. The conditions varied in the experiments were type of vehicle behavior (door opening vs. reversing), method used to display the sign (animated vs. flashing), and the timing of displaying the sign. Our results showed that the participants were aware of the seriousness of avoidance, regardless of age, but the effect of timing on avoidance behavior varied according to age.

Due to improvements in projection technology, dynamic signs are now becoming popular for various applications, such as guidance for to users in public facilities (DesignCollector, 2012; Aledo, 2017; YouTube, 2013; Ecolight, 2016), information for drivers (Landscape + Urbanism, 2010; Laservision, n.d.), and warning pedestrians away from vehicles (TechCrunch, 2015). Currently, however, methods have not been standardized, particularly with respect to visibility, accessibility, effects on behavior, and expressiveness. These issues should be discussed to avoid haphazard signage.

\section{Acknowledgements}

We thank Shingo Terada and Hiroko Ojima from RTC and the Human Informatics Research Institute at AIST for their assistance in analyzing the research data. The present study was conducted under the "2016FY Japanese Industrial Standards development project toward floor lighting (Japanese Standards Association)" as part of the project for developing high-function Japanese Industrial Standards, commissioned by Ministry of Economy, Trade and Industry (METI).

\section{References}

Adachi, Y., Harada, E., Suto, S., Kumada, T., \& Fujiwara, T. (2014). Cognitive Aging and Usage of Novel Artifact: A Database Analysis of the Center for Usability and Aging Research (CUAR) on a Usability Testing of Automatic Coffeemaker. Cognitive Studies, 21, 83-99. (In Japanese)

Aledo. (2017). Projection of Light Symbols. http://en.aledo.cz/projection-of-light-symbols-and-signs

Balk, S. A., Bertola, M. A., \& Inman V. W. (2013). Simulator Sickness Questionnaire: Twenty Years Later. Proceedings of the Seventh International Driving Symposium on Human Factors in Driver Assessment, Training, and Vehicle Design, USA, 257-263.

Brown, I. D., \& Groger, J. A. (1988). Risk Perception and Decision Taking during the Transition between Novice and Experienced Driver Status. Ergonomics, 31, 585-597.

Cycle Street City Multi-Purpose Virtual Cycling System. (2016). http://flovel-sports.jp/technosports/cycle/cyclestreet_city/

Design Collector. (2012). Interactive Navigation by SILA. http://designcollector.net/likes/interactive-navigation-by-sila

Ecolight. (2016). Projected Floor Marking. http://www.gobo-projector.eu/news/february-18th-2016

Folstein, M. F., Folstein, S. E., \& McHugh, P. R. (1975). Mini-Mental State, a Practical Method for Grading the Cognitive State of Patients for the Clinician. Journal of Psychiatric Research, 12, 189-198. 
Institute for Traffic Accident Research and Data Analysis. (2015). Traffic Accident Analysis Report (No. 115). Tokyo. (In Japanese)

Institute for Traffic Accident Research and Data Analysis. (2016). Traffic Accident Analysis Report (No. 114). Tokyo. (In Japanese)

Jackson, G. R., Owsley, C., \& McGwin, G. Jr. (1999). Aging and Dark Adaptation. Vision Research, 39, 3975-3982.

Kennedy, R. S., Lane, N. E., Berbaum, K. S., \& Lilienthal, M. G. (1993). Simulator Sickness Questionnaire (SSQ): A New Method for Quantifying Simulator Sickness. International Journal of Aviation Psychology, 3, 203-220. https://doi.org/10.1207/s15327108ijap0303_3

Landscape + Urbanism (2010). Smart Wayfinding. http://landscapeandurbanism.blogspot.jp/2010/03/smart-wayfinding.html

Laservision (n.d.). Activated 8 Times in 8 Weeks, with 100\% Success! http://www.laservision.com.au/portfolio/softstop/

Sakata, R., Matsubara, T., Watanabe, H., Ito, N., \& Ujike, H. (2016). Visibility of " $D y$ namic Signs" Used to Signal Open Vehicle Doors and Their Effect on Avoidance Action. Poster Session Presented at the 6th International Conference for Universal Design, Nagoya.

Shiota, K., \& Ikeda, M. (2008). Influence of Aging Change in Visual System on Postural Control. Journal of Japan Academy of Health Sciences, 11, 145-152. (In Japanese)

Suto, S., Kumada, T., Kitajima, M., \& Sato, T. (2008). Relationship between Cognitive Functions and Usage of IT-Based Equipment. Poster Session Presented at the 2008 Cognitive Aging Conference, Atlanta.

TechCrunch (2015). Google Granted Patent That Enables Self-Driving Cars to Interact with Pedestrians.

https://techcrunch.com/2015/11/30/google-granted-patent-that-enables-self-driving-ca rs-to-interact-with-pedestrians/

Ujike, H., \& Sagawa, K. (2003). CSF as a Function of Age and Its Application to Visibility of Letters. In Proceedings of the CIE Symposium'02 on Temporal and Spatial Aspects of Light and Color Perception and Measurement (Vol. 25, pp. 81-87).

YouTube (2013). Navigation Moving Gobo Projection, Hotel Hilton, Prague. https://www.youtube.com/watch?v=K1Cr3DJBS4I

\section{Submit or recommend next manuscript to SCIRP and we will provide best} service for you:

Accepting pre-submission inquiries through Email, Facebook, LinkedIn, Twitter, etc. A wide selection of journals (inclusive of 9 subjects, more than 200 journals)

Providing 24-hour high-quality service

User-friendly online submission system

Fair and swift peer-review system

Efficient typesetting and proofreading procedure

Display of the result of downloads and visits, as well as the number of cited articles

Maximum dissemination of your research work

Submit your manuscript at: http://papersubmission.scirp.org/

Or contact psych@scirp.org 